\title{
Robótica educacional: Uma ferramenta para ensino de lógica de programação no ensino fundamental
}

\author{
Manassés Fernandes ${ }^{1}$, Camila A. M. dos Santos ${ }^{1}$, \\ Edmar E. P. de Souza ${ }^{1}$, Marcos Guimarães Fonseca ${ }^{1}$ \\ ${ }^{1}$ Centro Universitário Ruy Barbosa Wyden \\ Caixa Postal 41940-320 - Salvador - BA - Brasil \\ manasses.fernandes@outlook.com, marcos.fonseca@areal.edu.br \\ camila.santos@frb.edu.br, edmar.souza@frb.edu.br
}

\begin{abstract}
Educational Robotics has, over the years, become a tool of technological practice that presents dynamism and motivation to the teaching and learning process. The experience of various situations and practical activities motivate the students to solve problems, work in groups, critical capacity, development of logical reasoning, so that he becomes a protagonist in the construction of his knowledge. This article aims to report the experience of a student of the Control and Automation Engineering course, in the promotion of educational activities and development of strategies for the teaching of programming logic with the use of educational robotics in private schools in Salvador-BA with students level elementary II.
\end{abstract}

Resumo. A Robótica educacional vem, ao longo dos anos, se concretizando como uma ferramenta de prática tecnológica que apresenta dinamismo e motivação ao processo de ensino e aprendizagem. A vivência de diversas situações e atividades práticas motivam os alunos para a resolução de problemas, trabalhos em grupos, capacidade crítica, desenvolvimento do raciocínio lógico, de maneira que ele se torna protagonista na construção do seu conhecimento. Este artigo tem como objetivo relatar a experiência de um estudante do curso de Engenharia de Controle e Automação, na promoção de atividades educacionais e desenvolvimento de estratégias para o ensino de lógica de programação com uso da robótica educacional em escolas da Rede privada de ensino em Salvador- BA com alunos de Ensino Fundamental II.

\section{Introdução}

O aumento considerável do uso de dispositivos eletrônicos nas escolas vem demonstrando quanto o avanço tecnológico se faz presente no cotidiano, tornando mecanismos indispensáveis, uma vez que as crianças estão cada vez mais imersas em um ambiente tecnológico e digital. Neste contexto surge o ensino da robótica educacional, que tem como seu principal instrumento o uso de robôs, permitindo aos alunos construir e programar sistemas robóticos para auxiliar e estimular melhorias no processo de ensino e aprendizagem. Segundo [Castilho 2002] a robótica educacional tem como objetivo disponibilizar um ambiente que seja possível o aluno aprender e não apenas manipular um robô, mas saber todos os conceitos lógicos de forma estimular o processo de raciocínio e criatividade. 
VII Congresso Brasileiro de Informática na Educação (CBIE 2018)

Anais do XXIV Workshop de Informática na Escola (WIE 2018)

Os algoritmos estão sendo utilizados de forma invisíveis em momentos do dia-adia em que realizamos atividades e resolução de problemas, sendo conjuntos de passos finitos e organizados para resolver determinada problemática. Em [da Silva 2015] afirmase que a Estônia e o Reino unido adotaram nas programações dos currículos escolares, visualizando a necessidade para o mercado de trabalho, bem como muitos países já vêm utilizando o ensino de algoritmos computacionais e lógica de programação.

Começando enquanto crianças se tem um grande ganho ao aprender lógica de programação [GATES 2014]. A prática em escrever esses programas resulta na ampliação da capacidade de raciocínio e auxilia o pensamento em todas as outras áreas. Seguindo esse discernimento, este artigo tem como objetivo expor a experiência vivenciada por estudantes do curso de bacharelado em engenharia de controle e automação na promoção de atividades educacionais no desenvolvimento de estratégias para o ensino de lógica de programação com uso da robótica em escolas de nível fundamental.

\section{Robótica educacional}

Podendo ser definida como o conjunto de conceitos tecnológicos aplicados à educação, a robótica educacional permite aos alunos o acesso a computadores, softwares, componentes mecânicos e eletrônicos, além de uma interface de programação para integração e funcionamento dos equipamentos e dispositivos, somado ao uso de recursos pedagógicos para a construção de um ambiente escolar favorável ao aprendizado [Pirola 2010].

A robótica estimula o desenvolvimento do conhecimento através de situações que fazem o indivíduo pensar em soluções, de forma relacionar a teoria com a prática utilizando o que aprendeu em sala de aula e em experiências anteriores, para resolver determinados problemas. Segundo [Jr et al. 2008] a construção de robôs na escola proporciona aos professores e alunos a ensinar, aprender, descobrir, inventar em processos coletivos, capazes de conectar abstração e mundo concreto. Além disso, pode associar conteúdo, bem como estimular a criatividade e participação por meio do trabalho em grupo e a resolução de problemas no cotidiano escolar.

A robótica educacional disponibiliza ferramentas e aumenta o número de atividades que podem ser desenvolvidas, permitindo a interdisciplinaridade e expandindo o ambiente de aprendizado através da vivência do método científico somada a prática [do Rocio Zilli 2004].

\subsection{O Ensino de Lógica de Programação com a robótica}

A lógica de programação é a ordem da razão e comunicação para programação de computadores, podendo ser trabalhadas em várias linguagens e estruturas conhecidas como algoritmos. Tal conceito corrobora para o seguimento do melhor rumo para a resolução de problemas [Forbellone 2005].

As dificuldades encontradas no estudo de lógica de programação, na maioria das vezes, fazem com que alunos desistam de cursos de computação por não conhecerem previamente os conteúdos específicos exigidos se tornando um ensino não trivial [Ferreira et al. 2010]. Levantando a importância da necessidade do ensino de conceitos computacionais desde o ensino básico, com foco principal na lógica de programação, 
VII Congresso Brasileiro de Informática na Educação (CBIE 2018)

Anais do XXIV Workshop de Informática na Escola (WIE 2018)

já que a mesma pode dar auxílio no desenvolvimento do aprendizado das crianças [da Conceição Araújo et al. 2015].

[Rapkiewicz 2006] defende que a falta de método de ensino e material didático inovador são barreiras para a aprendizagem, uma vez que essas ferramentas possibilitam a transformação do que é abstrato em concreto. A maioria das instituições passa o conteúdo nas formas convencionais sem privilegiar os meios para aprendizagem, trazendo a necessidade de ações pedagógicas para complementar o processo que permita o desenvolvimento de fatores como a atenção, concentração, criatividade e habilidades que ajudam no raciocínio.

O utilizar ferramentas lúdicas é possível atrair a atenção dos alunos, o que por sua vez permite o aprendizado e desenvolvimento de habilidades com mais facilidade, tornando as atividades mais prazerosas e desafiadoras, sem limite de possibilidades e uma dimensão simbólica [de Macedo and Petty 2005].

Imagine uma criança que constrói o seu próprio brinquedo e, melhor ainda, ela adora brincar com ele porque é bonito e tecnológico, o bônus é que ela aprende muito enquanto o constrói e programa. Esse é apenas um dos benefícios de ensinar robótica para crianças. Em diversos países do mundo o ensino da robótica já faz parte da matemática e física, essa prática corrobora para o desenvolvimento de várias outras habilidades como pensamentos mais específicos, lógicos e práticos [HAPPYCODE 2016].

Para a construção de um equipamento robótico é necessário a combinação de diversos conceitos e conhecimentos de diferenciadas áreas, as atividades devem ocorrer de forma produtiva, principalmente trabalhadas por grupos de alunos, encaixando no conceito de transdisciplinaridade como a valorização do conhecimento compartilhado na construção de algo novo resultado da soma de conhecimentos de cada indivíduo [do Rocio Zilli 2004].

Ao aliar o ensino de lógica de programação com a robótica, é possível desenvolver o raciocínio e assimilar o conteúdo ministrado. De acordo com [Castilho 2002] "a teoria de equilibração", de PIAGET , trata de um ponto de equilíbrio entre a assimilação e a acomodação, e assim é considerado como um mecanismo auto regulador na interação com o meio-ambiente. Na robótica educacional é oferecido ao aluno um ambiente onde o mesmo pode manusear, criar, programar por si mesmo e, através desta prática lúdica, desenvolve o raciocínio lógico tão importante nas diversas áreas do conhecimento.

\section{Materiais e Métodos}

Este trabalho foi formulado levando em consideração os conceitos metodológicos da pesquisa-ação, por meio de atividades com aplicações da robótica educacional sendo necessário a troca de informações entre os participantes das oficinas e o instrutor.

De acordo com [?], a pesquisa-ação é mais independente e objetiva quando comparada a pesquisa tradicional, uma vez que ela une os fundamentos teóricos à prática. Normalmente é uma estratégia usada em situações que tem como objetivo melhorar a compreensão na aplicação dos conceitos.

Seguindo a metodologia, foi levado em conta uma determinada ordem de procedimentos para a resolução de uma situação problema, a fim de estimular práticas em sala de aula a envolver atividades do dia a dia na busca de novas experiências no processo 
VII Congresso Brasileiro de Informática na Educação (CBIE 2018)

Anais do XXIV Workshop de Informática na Escola (WIE 2018)

de aprendizagem, utilizando recursos estruturais das escolas e materiais de uso para a robótica.

A robótica educacional para o ensino fundamental tem como objetivo desenvolver a capacidade lógica com boa interpretação e organização de forma a resolver problemas, além do desenvolvimento de relação no trabalho em grupo.

Para o desenvolvimento de lógica de programação foram utilizados fundamentos de algoritmos computacionais, de maneira a expor conceitos de sintaxes e semântica, operadores lógicos e instruções de entrada e saída que posteriormente montadas e testadas no software Modelix.

O Software Modelix System consiste em uma interface de desenvolvimento para controle de hardware Arduino. Este software é baseado na transformação de linguagem simples de programação, feita com a utilização de fluxogramas, como principal ferramenta da construção da lógica e do programa a ser executado no Arduino.

O fluxograma é uma ferramenta em forma de diagrama que utiliza notação gráfica de simples entendimento e utilização na representação visual de um algoritmo, possibilitando a visualização detalhada de todos os passos e etapas a serem executadas em um código. Após a construção do diagrama o software, de forma automática, transforma-o em linguagem computacional e envia para o Arduino.

As atividades foram trabalhadas com uso de dinâmica em grupo, levando os integrantes despertar habilidade de comunicação para resolução de problemas entre outros.

\section{Resultados - Relatos de Experiência}

Inicialmente os estudantes tiveram uma demonstração de forma lúdica do funcionamento de alguns dispositivos e robôs em diferentes aplicações. Em seguida foi proposto a separação em grupos para montagem de uma placa eletrônica em formato de robô (figura 1), onde alguns componentes como LEDS (Diodo emissor de luz), resistores e BUZZER dariam forma ao robô, que caso fossem instalados corretamente a partir das instruções dadas dentro do tempo as equipes seriam premiadas com um brinde.

No segundo encontro as atividades foram relacionadas a construção de algoritmos exercitando o raciocínio lógico dos alunos em situações cotidianas, como a construção de passo a passo de atividades do dia-a-dia e resolução de problemas matemáticos simples.

No encontro seguinte os estudantes tiveram a demonstração de conceitos de eletrônica básica, robótica, placa micro controladora Arduino e a utilização de componentes básicos, como o controle de um Led através do Arduino com programação previamente carregada em seu microprocessador.

O quarto encontro teve como objetivo iniciar a construção de um robô que desvia de obstáculos, com o intuito de estimular a imaginação dos alunos dando-lhes a possibilidade da criação e customização de um robô criado totalmente por eles, do nome a forma. Para construção e conclusão das estruturas dos projetos os alunos usaram bastante imaginação tanto na composição dos robôs quanto na utilização de materiais encontrados em casa de forma a comportar a placa micro controladora Arduino, protoboard, dois servos motores e um sensor ultrassônico.

$\mathrm{Na}$ quinta semana iniciaram as atividades de lógica de programação exercitando 
VII Congresso Brasileiro de Informática na Educação (CBIE 2018)

Anais do XXIV Workshop de Informática na Escola (WIE 2018)

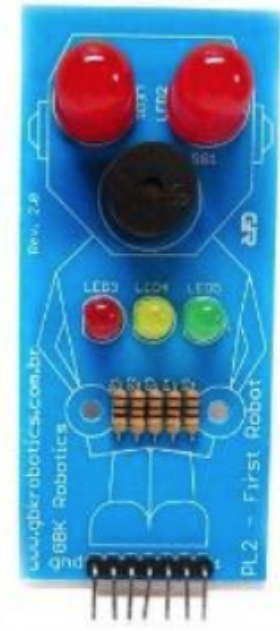

Figura 1. Robô GBK

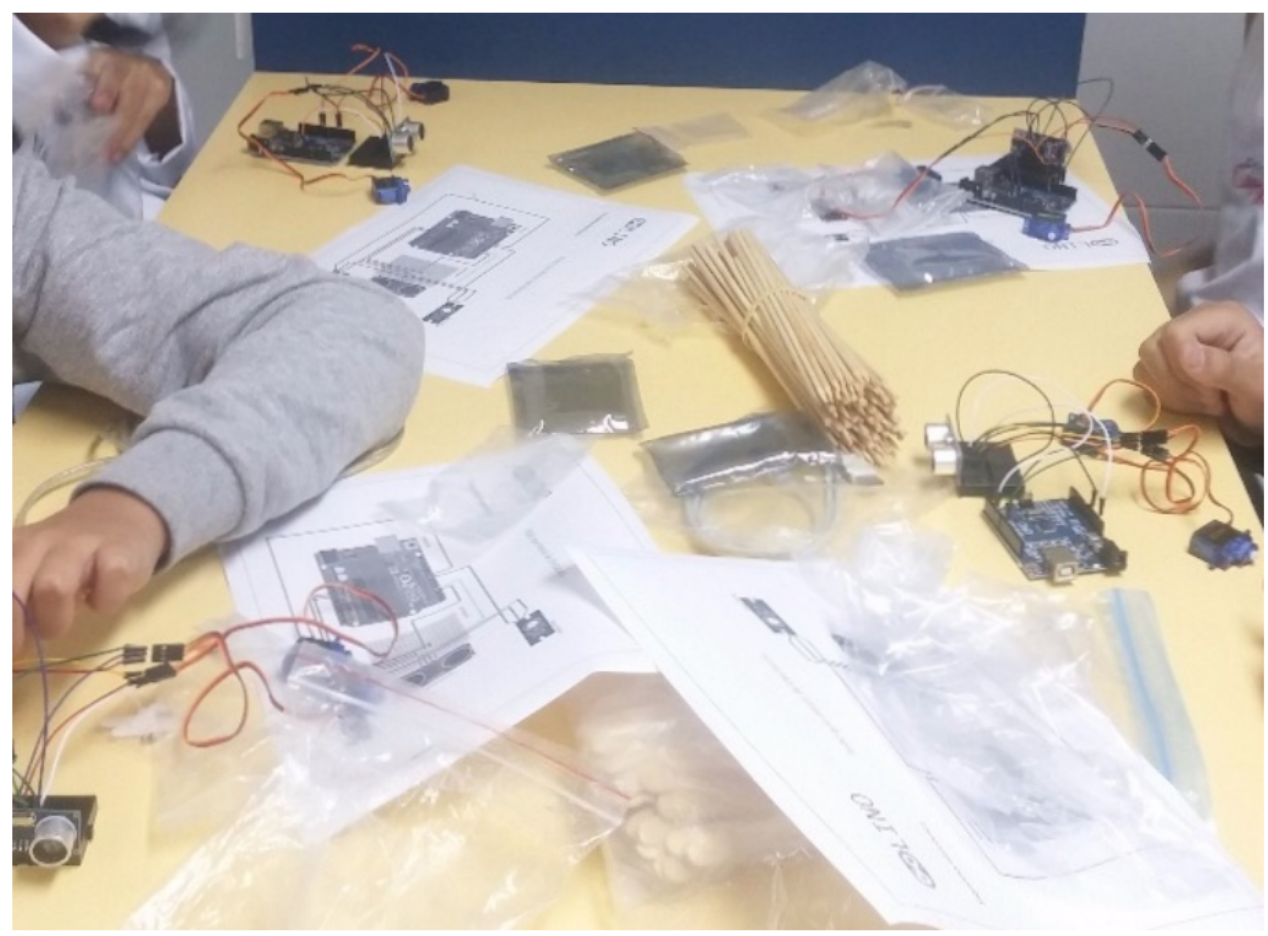

Figura 2. Realização de atividade para construção de robôs. 
VII Congresso Brasileiro de Informática na Educação (CBIE 2018)

Anais do XXIV Workshop de Informática na Escola (WIE 2018)

o que já tinha sido trabalhado nos primeiros encontros, desta vez com foco na utilização de fluxogramas na construção dos algoritmos, visando a ambientação com o software Modelix.

A partir da apresentação do software e da linguagem de programação utilizada, foi dada a atividade para construção da lógica em fazer o LED piscar a cada um segundo. Para a triagem de fixação do conteúdo foi realizado a solução na lousa do exercício supracitado com a participação dos estudantes. Posteriormente foi lançado um desafio para as equipes de montar o fluxograma no Modelix à ser enviado para o Arduino e modificado à medida fossem concluindo. Tal dinâmica foi de fundamental importância para o manuseio e reconhecimento das ferramentas básicas para construção dos algoritmos seguintes.

Com avanço das atividades os encontros seguintes foram realizados no mesmo sentido para utilização de sensor para controle de atuadores, inicialmente sendo utilizado em conjunto sensor ultrassônico e LED, partindo posteriormente para controle de motores.

Com as instruções executadas, os alunos tiveram o desafio de montar a lógica de programação para tornar os respectivos robôs autônomos, de forma separada os conceitos básicos e lógicas necessárias para automatização foram trabalhadas para que os alunos pudessem ter uma carga de conhecimento prévio para alcançar o objetivo, cabendo a eles a montagem de uma lógica que fizesse a junção entre os algoritmos criados em sala.

Como esperado as equipes trabalharam em conjunto, fazendo umas séries de discussões e questionamentos entre os membros, realizando esboços de códigos baseados nos resultados do raciocínio comum gerado na equipe. Após a criação de um conceito para a lógica a programação, a mesma foi construída no software enviadas para os robôs montados por eles. Ao perceber a necessidade de ajustes os estudantes foram estimulados a procurar os problemas na programação ou realizar ajustes finos para o bom funcionamento de seus protótipos.

No decorrer das atividades foi perceptível o empenho dos alunos movido pelos desafios em equipe, onde a troca de informações e funções foram divididas entre eles, de forma que as equipes concluíram as atividades propostas com sucesso, levando ao entendimento que os participantes se sentiram capazes de fazerem seus próprios equipamentos robóticos.

Os resultados foram satisfatórios, todas as lógicas de programação foram criadas a partir da discussão entre os membros da equipe, assim como os ajustes realizados após os primeiros testes com os robôs. Cada robô teve uma forma de comportamento diferente, mas executando a atividade proposta que seria o desvio de sua rota a partir da localização de um obstáculo à frente.

\section{Conclusões e Considerações Finais}

A dinâmica do processo de aprendizado e criação de projetos apresenta uma proposta interdisciplinar com atribuição ao desenvolvimento da criatividade e pensamento crítico, aflorando habilidades dos estudantes em frente a situações problemas, com utilização de algoritmos criados através da lógica de programação, visando estabelecer uma sequência de instruções para resolver determinado problema, levando o indivíduo a pensar e transformar suas atividades em um algoritmo com procedimentos, entrada e 
VII Congresso Brasileiro de Informática na Educação (CBIE 2018)

Anais do XXIV Workshop de Informática na Escola (WIE 2018)

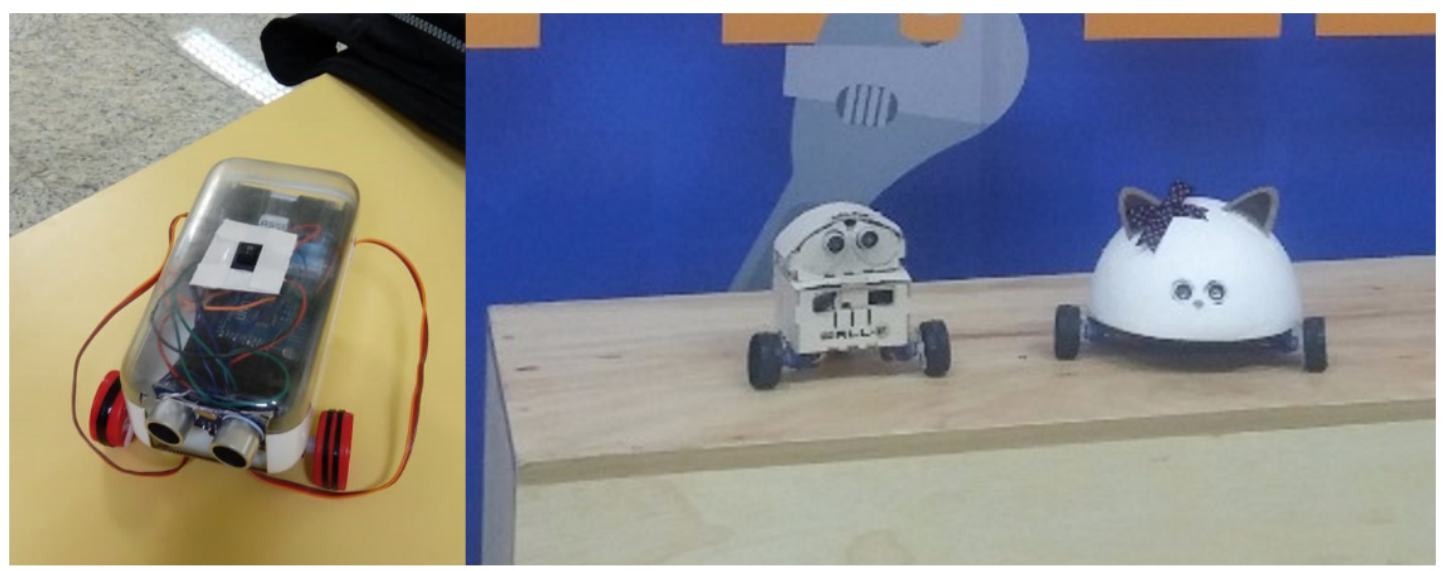

Figura 3. Exemplares dos robôs produzido nas aulas.

saída de informações para determinada ação.

As técnicas utilizadas para o estímulo da busca de conhecimento dos alunos foram positivas, visto que a construção de um robô de total autoria das equipes possibilitou troca de informações, onde cada estudante teve um papel fundamental pondo em prática suas respectivas habilidades, para estabelecer uma relação da teoria aplicada na lógica de programação e conceitos básicos de eletrônica com a prática direcionadas em seus próprios robôs.

O maior desafio foi na transformação de conceitos técnicos e teóricos relacionados a lógica de programação em exemplos de fácil entendimento, uma vez que foi observado no decorrer das aulas teóricas maior dificuldade em assimilar os conteúdos.

Ao aplicar o conteúdo à prática foi perceptível a capacidade de abstração na construção dos programas, executando os conceitos teóricos sem demonstrar relutância ao operar o ambiente de programação do software Modelix, por possuir um visual simples e sendo operado apenas com formas geométricas utilizadas nos conceitos aplicados em aulas.

A utilização dos ensinamentos da robótica possibilitou transmitir de maneira simples e lúdica conceitos de disciplinas básicas de lógica de programação e outras áreas como física e matemática, permitindo aos alunos desenvolver conhecimentos a serem utilizado em situações cotidianas.

Diante desse relato espera-se que o mesmo possa servir de fonte de inspiração e auxiliar na disseminação da robótica educacional como estratégias para melhorias no processo de ensino e aprendizagem. E assim os conteúdos julgados como de difícil compreensão na educação básica sejam desmistificados e sejam vistos como fáceis, acessíveis e interessantes, de maneira que os alunos se sintam mais confiantes para desafios futuros.

\section{Referências}

Castilho, M. I. (2002). Robótica na educação: Com que objetivos? Monografia de Especialização em Informática na Educação. Universidade Federal do Rio Grande do Sul/Porto Alegre. 
VII Congresso Brasileiro de Informática na Educação (CBIE 2018)

Anais do XXIV Workshop de Informática na Escola (WIE 2018)

da Conceição Araújo, D., Rodrigues, A. N., de Araújo Silva, C. V., and Soares, L. S. (2015). O ensino da computação na educação básica apoiado por problemas: Práticas de licenciados em computação. Anais do XXIII WEI (Workshop sobre Educação em Computação).

da Silva, C. (2015). Introdução ao ensino de lógica de programação para crianças do ensino fundamental com a ferramenta scratch. Dissertação (Núcleo De Educação à Distância Licenciatura em Informática)- Universidade Federal De Roraima.

de Macedo, L. and Petty, A. L. S. (2005). Os Jogos e o Lúdico na Aprendizagem Escolar, volume 1. Artmed.

do Rocio Zilli, S. (2004). A robótica educacional no ensino fundamental: pespectivas e práticas. santa catarina. Dissertação (Mestrado), Programa de Pós?Graduação em Engenharia de Produção, Universidade Federal de Santa Catarina.

Ferreira, C., Gonzaga, F. B., and dos Santos, R. P. (2010). Um estudo sobre a aprendizagem de lógica de programação por demonstração. Anais do XVI WEI (Workshop sobre Educação em Computação).

Forbellone, A. L. V. (2005). Lógica de programação: a construção de algoritmos e estruturas de dados, volume 1. Prentice Hall.

Jr, V. L., Pinto, L., and da Silva, V. J. (2008). A robótica como ambiente de programacção utilizando o kit lego mindstorms. Simpósio Brasileiro de Informática na Educação XIX SBIE.

Pirola, N. A. (2010). Ensino de ciências e matemática, IV: temas de investigação, volume 1. UNESP.

Rapkiewicz, C. E. (2006). Estratégias pedagógicas no ensino de algoritmos e programação. Revista Novas Tecnologias na Educação - RENOTE. 